\title{
From fused aromatics to graphene-like nanoribbons: The effects of multiple terminal groups, length and symmetric pathways on charge transport
}

\author{
Ante Bilić* \\ CSIRO Mathematics, Informatics and Statistics, Private Bag 33, Clayton South VIC 3169, Australia \\ Julian D. Gale \\ Nanochemistry Research Institute, Department of Chemistry, Curtin University, P.O. Box U1987, Perth, WA 6845, Australia \\ Stefano Sanvito \\ School of Physics and CRANN, Trinity College, Dublin 2, Ireland
}

(Received 24 August 2011; published 17 November 2011)

\begin{abstract}
A class of molecular ribbons, with almost-ideal charge transmission, that is weakly dependent on the anchoring structure or electrode crystalline orientation and easy to synthesize has been identified. Charge transport through two sets of aromatic nanoribbons, based on the pyrene and perylene motifs, has been investigated using density functional theory combined with the nonequilibrium Green's function method. The effects of wire length and multiple terminal thiolate groups at the junction with gold leads have been examined. For the oligopyrene series, an exponential drop in the conductance with the increase of the wire length is found. In contrast, the oligoperylene series of nanoribbons, with dual thiolate groups, exhibits no visible length dependence, indicating that the contacts are the principal source of the resistance. Between the $\mathrm{Au}(001)$ leads, the transmission spectra of the oligoperylenes display a continuum of highly conducting channels and the resulting conductance is nearly independent of the bias. The predictions are robust against artefacts from the exchange-correlation potential, as evidenced from the self-interaction corrected calculations. Therefore, oligoperylene nanoribbons show the potential to be the almost-ideal wires for molecular circuitry.
\end{abstract}

DOI: 10.1103/PhysRevB.84.205436

\section{INTRODUCTION}

Over the past decade, charge-transport properties of single molecules have attracted a great deal of interest because of the need for ever-increasing circuit miniaturization and the limits that the conventional metal-oxide-semiconductor technology faces. Conjugated aromatic molecules, such as polycyclic hydrocarbons, ${ }^{1-3}$ and oligomers, such as phenylene-vinylene, ${ }^{4}$ represent some of the most frequently investigated molecular wires. Despite the large body of conductivity studies found in the literature, a class of wires with transport properties suitable for molecular circuit usage has not yet been identified. An ideal class of molecular wires needs to provide a high and consistent level of conductance over a wide bias region and for various lengths of the molecules. Due to the experimental isolation of graphene in 2004 (Ref. 5) and the prospects it offers because of its very low resistance, there has been an increasing effort toward harnessing this novel material in nanoelectronics. Graphene is a two-dimensional material, which owes its desirable charge-transport properties to the honeycomb lattice of $s p^{2}$ hybridized carbon atoms, and can be viewed as an infinitely large aromatic molecule. While there exists no one-dimensional analog of graphene consisting of carbon alone (nanotubes are two-dimensional, nonplanar materials, although considered quasi-one-dimensional from the transport point of view), one can think of linear acenes as its closest one-dimensional counterpart. Benzene, the prototypical component of molecular wires, could then be formally assigned the zeroth dimension, like a quantum dot. The smallest nonlinear acene molecules are phenanthrene and pyrene, with three and four rings in the plane, respectively. Charge transport through both of them has been investigated
PACS number(s): 85.65.+h, 71.15.Mb, 72.80.Le, 72.80.Vp

theoretically in a study by Cohen et al. that focused on the effects of molecular conjugation, length, and electrode coupling on the transport over a range of polycyclic aromatic molecules. ${ }^{1}$ Subsequently, Liu et al. studied the effects of multiple electron pathways in linear polyacenes and a class of molecules based on biphenyl dithiol, the latter also involving phenanthrene and pyrene. ${ }^{2}$ Shortly after, they extended this work to include the oligomers of those molecules, as well as those of thiophene, pyrrole, and porphirine. ${ }^{3}$ Those studies were based on the $\mathrm{Au}(111)$ model of leads-whose symmetry is not compatible with that of the molecules-because the focus was on the transport pathways through the wire, rather than across the entire junction.

Here we report computational results on the conductance through two classes of aromatic nanoribbons, namely, oligopyrenes and oligoperylenes, as a function of the wire length. The two series share the same scaffolding of alternating double and single rings along the transport axis, armchair edge, and $C_{2 v}$ symmetry. The pyrene and perylene molecules, with thiol linker groups, are shown in Fig. 1. While oligopyrenes terminate with single phenyl rings, oligoperylenes are terminated with double rings and, hence, dual thiol head groups are proposed as the linkers at both ends of these wires. A trend of decreasing conductance with increasing length has been observed in the case of the oligopyrene series, with conventional single thiolate contacts at both terminals. In contrast, the nanoribbons from the oligoperylene series, with dual thiolate terminal groups, exhibit a linear current-voltage relationship over a large bias range, with a very high conductivity, which is seemingly independent of the wire length. Hence, these have emerged as the best candidates for use as wires in molecular circuits. 


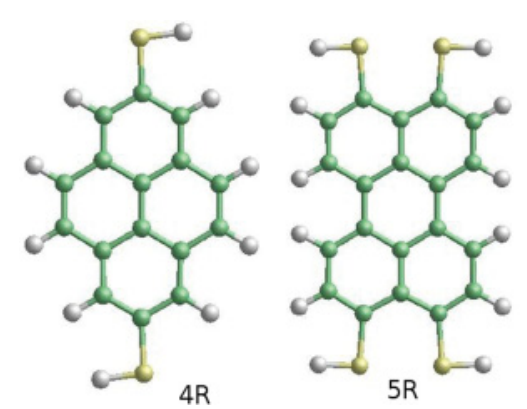

FIG. 1. (Color online) The $4 \mathrm{R}$ (pyrene dithiol) and 5R (perylene tetrathiol) wires.

\section{COMPUTATIONAL METHODOLOGY}

In the present work, the computational approach taken closely follows that previously validated in the study of transport through an oligo-phenylene-vinylene molecular wire. ${ }^{4}$ The important differences are the use of both the $\mathrm{Au}(001)$ and $\mathrm{Au}(111)$ surfaces for the metal contacts and the local density approximation (LDA) ${ }^{6}$ in the present study versus only the conventional $\mathrm{Au}(111)$ surface and the generalized gradient approximation (GGA) considered previously. The addition of the (001)-oriented gold substrate has been dictated by the aim of investigating the effects of the symmetric transport pathways on the overall conductance across the junction. Oligoperylene wires comprise dual thiol groups at symmetric points. Given the known tendency of thiols to strongly and nonselectively bind to gold on the hexagonal (111) surface, ${ }^{7}$ the symmetry of which is different to that of the considered wires, a large number of adsorbate conformations with similar energies would be possible. The investigation of the transport across even a subset of these would make this work intractable. In contrast, the plane symmetry and $A B A B$ layer stacking of the (001) surface allowed for a limited number of highly symmetric, energetically favorable atomic structures of the extended molecule. The LDA exchange-correlation functional was chosen for two reasons. First, the LDA was employed both in the work of Cohen et al. ${ }^{1}$ and Liu et al., ${ }^{2,3}$ and given the potential for direct comparison between these earlier studies and the current work, we have strived to minimize possible variances in the results which arise simply due to differences in the methodology. Second, since neither the LDA or GGA can predict a priori the band alignment between the leads and wire with the necessary accuracy, 8,9 there would be no reason to prefer either class of functional in this regard. However, the important difference is that self-interaction correction (SIC) can be applied in combination with the LDA to the scattering region of the junction to partly rectify the artefacts arising from this misalignment. ${ }^{10-12}$ In the present work, this has been done by utilizing the atomic self-interaction correction $(\mathrm{ASIC})^{13}$ for several representative calculations. This approach allows us to conclude that the conductivity of the investigated ribbons is a genuine property and not an artefact of the exchange-correlation functional utilized.

Single-point energy calculations and geometry optimizations were performed using density-functional theory (DFT) as implemented in the SIESTA program. ${ }^{14}$ The SIESTA method used a linear combination of atomic orbitals (LCAO) to represent the Kohn-Sham eigenstates. Core electrons and nuclei are accounted for by norm-conserving pseudopotentials in the Kleinman-Bylander fully separable form. ${ }^{15}$ The modified scheme of Troullier and Martins ${ }^{16}$ was utilized to generate pseudopotentials for $\mathrm{H}, \mathrm{C}, \mathrm{F}, \mathrm{S}$, and $\mathrm{Au}$, with relativistic corrections included for $\mathrm{S}$ and $\mathrm{Au}$. The one-electron Kohn-Sham eigenstates were expanded in a basis of strictly localized, ${ }^{17}$ numerical, pseudo-atomic orbitals. ${ }^{18}$ Basis functions were obtained by evaluating the eigenfunctions of the isolated atoms confined within a sphere. The range of the atomic orbitals was evaluated from an energy increase of $1 \mathrm{mRy}$ arising due to the spherical confinement, as opposed to $7 \mathrm{mRy}$ in the previous GGA-based study. ${ }^{4}$ The smaller energy shift was chosen so as to reduce the effects of confinement, which serve to reinforce the tendency of LDA to overbind, thereby yielding an improved gold lattice constant and optimized bond lengths with respect to the experiment. A split-valence scheme was employed to generate a single- $\zeta$ basis set for the $6 s$ and $5 d$ states of Au, and a double- $\zeta$ basis set for the $1 s$ state of $\mathrm{H}, 2 s$ and $2 p$ states of $\mathrm{C}$ and $\mathrm{F}$, and $3 s$ and $3 p$ states of $\mathrm{S}$, with a single- $\zeta$ shell of polarization functions for all five elements. An equivalent plane-wave cutoff of 350 Ry was used to determine the spacing of the mesh that SIESTA uses to evaluate energy and potential terms based on charge density.

Atomic geometries were relaxed via conjugate gradient optimization until the forces were smaller than $10 \mathrm{meV} \AA^{-1}$. However, of about a dozen junctions studied here, only the geometry of the smallest junctions, which comprise molecular wires made of one and two aromatic rings, was fully optimized. By using the atom coordinates from these structures, the other junctions were generated simply by the insertion of the inner benzene rings from the molecular geometries optimized in the gas phase, and increasing the junction gap accordingly to accommodate them. In this way, the computational effort is greatly reduced. While in this approach the residual forces are sizable, the effect of small wire distortions on the transport is rather minor. ${ }^{4}$ The generated geometries from the SIESTA computations, as well as all of the relevant computational parameters, were subsequently employed in electron-transport calculations. The latter were conducted using the nonequilibrium Green's function (NEGF) Landauer approach ${ }^{19}$ as implemented in the SMEAGOL package, ${ }^{20,21}$ which is interfaced to SIESTA.

Two models for the two-terminal device were considered. The first, described in detail in Ref. 4, is based on conventional $\mathrm{Au}(111)$ leads. Given the $C_{2 v}$ symmetry of the investigated nanoribbons, the junction of $\mathrm{Au}(001)$ terminated leads, with a compatible symmetry, has also been utilized. The (001) gold slab was 11-atomic-layers thick and the boundaries of the supercell were set up so that the single slab forms the two contacts, with five and six gold atomic layers on each side. The system was then partitioned into two leads and a scattering region. The four Au layers at the bottom and top of the cell (i.e., the inner eight layers of the slab) were the leads and their geometries were fixed to the bulk positions. The single (two) Au layer(s) below and above the vacuum region (i.e., the outer layers on each side of the slab) were taken as parts of the extended molecule with which they form the scattering region (this is the part treated at the NEGF self-consistent level). The atomic coordinates of these $\mathrm{Au}$ layers in the contact region were optimized for the junctions 
comprising the wires with one and two aromatic rings, and then the same coordinates were employed when the oligomers of those wires were used. The thickness of the vacuum, i.e., the separation of the electrodes, varied from $9.2 \AA$ for the two-ring molecular wire to $27.08 \AA$ for the thirteen-ring nanoribbon. A single molecule was inserted in the middle of the vacuum region, with $\mathrm{S}$ atoms coordinated either by the bridge sites or hollow sites.

The Au(001) surface was described by a square superstructure of eight gold atoms per layer. For the geometry optimizations, as well as for the transport computations, the Brillouin-zone integrations were performed on a $2 \times 2 \mathrm{k}$-point Monkhorst-Pack mesh in the plane of the surface. For the calculation of the bulk electronic structure of the gold leads, the reciprocal space was sampled on a $2 \times 2 \times 80$ grid, with the denser sampling in the direction of transport. Finally, for the optimizations of the gas-phase molecular wires in a large supercell, only the $\Gamma$ point was used. In the transport calculations, the complex part of the integral leading to the charge density is computed using 25 energy points on the complex semicircle, 20 points along the line parallel to the real axis, and 9 poles. The integral over real energies, necessary at finite bias, is evaluated over at least 500 points, ${ }^{20}$ and changes with bias in order to maintain a uniform mesh.

\section{RESULTS AND DISCUSSION}

\section{A. Conductance of nanoribbons between Au(111) terminals}

The $I-V$ characteristics and zero-bias transmission coefficients for pyrene (4R) and its seven-ring (7R) and ten-ring (10R) oligomers are shown in Fig. 2. The results for the transport through the prototypical molecular wire, namely, phenyl dithiol (PDT, or 1R hereafter), are also shown therein. It has been included in the oligopyrene set because it shares the single thiolate terminal groups with them and it can be considered to be the base member of the 1-4-7-10R series. The results for $1 \mathrm{R}$ and $4 \mathrm{R}$ are in very good agreement with those in Ref. 1, thus demonstrating the soundness of our computational approach. Low-bias conductance exhibits the familiar exponential decay with the length of wire. ${ }^{3}$ An attenuation factor $\beta$ of $0.25 \AA^{-1}$ is evaluated for this series, in reasonably good agreement with 0.38 obtained previously. ${ }^{3}$ The value of $\beta$ depends sensitively on the band alignment between the wire and terminals, which may differ between the two sets of calculations.

The $I-V$ characteristics and zero-bias transmission coefficients for perylene (5R) and its eight-ring (8R) and eleven-ring (11R) oligomers in the junction of (111) terminals are shown in Fig. 3, together with the results for naphthalene-1,4,5,8tetrathiol (labeled 2R). Similar to $1 \mathrm{R}$ and oligopyrenes, $2 \mathrm{R}$ has been included in the oligoperylene set because it shares the dual thiolate terminal groups with them and is considered to be the base member of the $2-5-8-11 \mathrm{R}$ series. The $I-V$ characteristics and zero-bias transmission coefficients demonstrate very favorable transport properties, which manifest themselves through a high current and a generally high transmission probability, often in the range between 1 and 2 . In addition, this series does not display a visible length dependence, apart from the $2 \mathrm{R}$ wire, which conducts somewhat better than
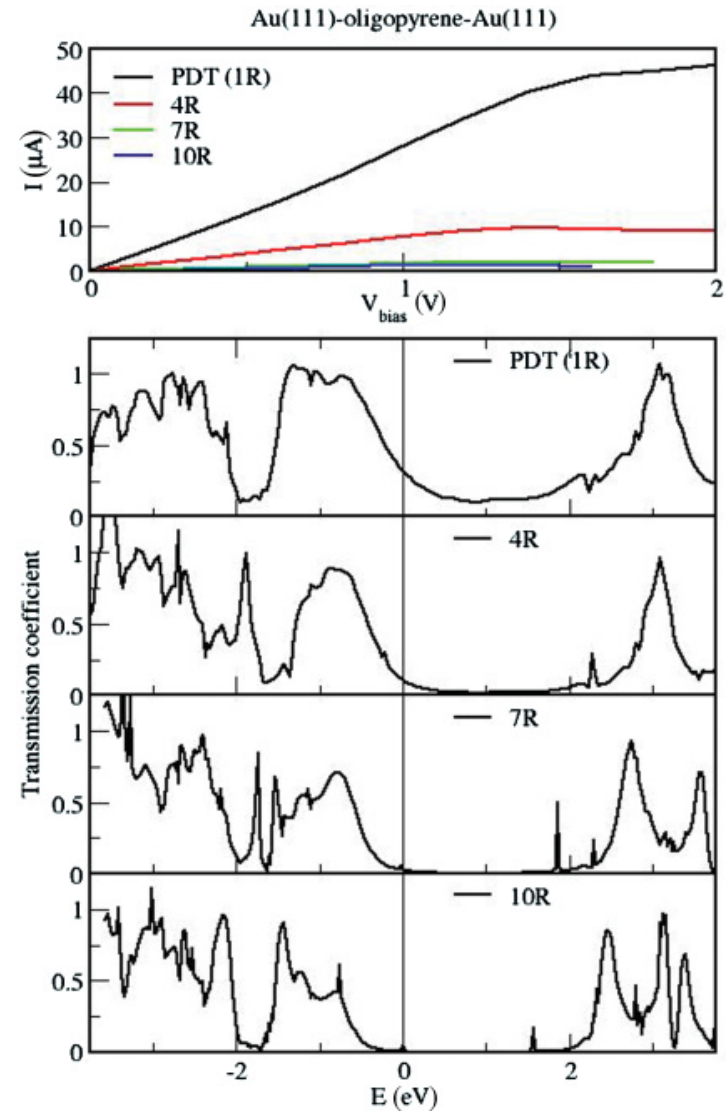

FIG. 2. (Color online) Upper panel: Current vs bias voltage for the 1-4-7-10R series of wires between the $\mathrm{Au}(111)$ terminals. Lower panels: The zero-voltage transmission probability across these junctions. The energy is given relative to the Fermi level.

the rest. The current through these molecules also exceeds twice that of the $1 \mathrm{R}$. There are several contributions to the superior transport properties of $2 \mathrm{R}$. First, the act of "fusing" two $1 \mathrm{R}$ molecules into the thiolated naphthalene gives rise to extra $\pi$ molecular orbitals (MOs) (owing to the additional 16 valence carbon electrons), whose energies fall between those of the highest occupied (HOMO) and lowest unoccupied (LUMO) molecular orbitals, which give rise to new conducting channels. Graphene and conjugated organic molecules are particularly good conductors because of these delocalized frontier orbitals with out-of-plane $\pi$ symmetry. Second, the new electronic state decreases the calculated HOMO-LUMO energy gap between from $3.25 \mathrm{eV}$ for $1 \mathrm{R}$ to $2.10 \mathrm{eV}$ for $2 \mathrm{R}$. Finally, the dual thiolate head groups provide a much stronger coupling between the terminals and carbon backbone (while also contributing to 12 extra valence sulfur electrons). The effective interaction is augmented across the entire junction (i.e., Au lead-S linkers-C backbone-S linkers-Au lead), giving rise to new frontier electronic states and a continuum of conducting channels that fill the transmission gap observed for $1 \mathrm{R}$. Consequently, the $2 \mathrm{R}$ junction exhibits even more desirable transport properties than the sum of two $1 \mathrm{R}$ junctions (or two 1R resistors connected in parallel). This effect is even more prominent in the case of pyrene (4R) and perylene (5R). Although of the same length, 5R has 11 out-of-plane $\pi$ MOs in the -8 to $-2 \mathrm{eV}$ energy range (i.e., about $\pm 3 \mathrm{eV}$ from 




FIG. 3. (Color online) Upper panel: Current vs bias voltage for the 2-5-8-11R series of wires between the $\mathrm{Au}(111)$ terminals. Lower panels: The zero-voltage transmission probability across these junctions. The out-of-plane $p$ orbital contribution to the partial density of states (PDOS) from an edge $\mathrm{C}$ atom is also shown for the $2 \mathrm{R}$ and $5 \mathrm{R}$ wires in the lower panel.

the Au Fermi level), and a calculated HOMO-LUMO gap of $1.33 \mathrm{eV}$. In contrast, 4R has two $\pi$ MOs less in the same range and a HOMO-LUMO gap of $2.31 \mathrm{eV}$. The formation of the additional frontier MOs in the more electron-rich oligoperylenes, compared to the oligopyrenes of the same length, is a consequence of the 16 extra valence carbon and 12 sulfur electrons. As a consequence, the gap between HOMO and LUMO is substantially lower in the oligoperylenes than in the oligopyrenes. For the former, the benefit of the additional conductance channels, owing to the extra $\pi$-type frontier MOs in a decreasing energy band, fully compensates for the penalty from having the longer wire.

An additional problem with the HOMO of the oligopyrenes is that because of its antisymmetry with respect to the long axis, it carries zero weight on the central atoms, including the terminal S atoms (the orbital plots are shown in Fig. 10 of Ref. 1 and Fig. 7 of Ref. 2). In the case of 4R, this results in the corresponding electronic state being completely decoupled from the gold contacts, without any energy dispersion across the Brillouin zone, and thus is nonconducting. As a result of the less favorable electronic structure, the $4 \mathrm{R}$ calculated current is about seven times lower than that of $5 R$. The trend quickly becomes very detrimental for oligopyrenes with increasing length. In contrast, for the more electron-rich oligoperylenes, the benefit of the additional $\pi$-type frontier MOs in a decreasing energy band seems to fully compensate for the penalty from having the longer wire. The projected density of states (PDOS) for the out-of-plane $p$ states on one of the edge $\mathrm{C}$ atoms, also shown in Fig. 3 for $2 \mathrm{R}$ and 5R, makes evident the contribution of the frontier $\pi$ states to the transmission probability. The peaks for both quantities arise at approximately the same energies. It is also evident that even the shallow peaks in the PDOS give rise to large amplitudes in the transmission spectrum if they are close to the Fermi level. Given the significance of the above findings, it is crucial to validate the calculations on representative wires from the 2-5-8-11R class by conducting ASIC transport calculations. The results for the 5R and $11 \mathrm{R}$ wires are also shown in Fig. 3. The correction causes a reduction in the current by a factor of $0.5-0.75$, but still gives a very high conductance. The corrected zero-bias transmission spectra display, as expected, larger gaps between occupied and unoccupied channels.

\section{B. Phenyl-dithiol adsorption and conductance between (001) contacts}

Since the adsorption of thiols onto the (001) gold surface has been much less investigated than on the (111) face, we have considered several possible coordination geometries of the phenylthiolate (PT) radical on $\mathrm{Au}(001)$. The attachment of the upright molecule (i.e., with the phenyl ring plane perpendicular to the surface) was investigated with the $S$ atom above the atop, fourfold hollow and bridge binding sites for two high-symmetry azimuthal orientations of the ring. In addition, flat-oriented attachments have also been considered (i.e., with the phenyl ring plane parallel to the surface). The most favorable adsorption, with a binding energy of $3.68 \mathrm{eV}$, is found to occur when the radical is oriented parallel to the plane of the surface, with the $\mathrm{S}$ atom above the bridge site, slightly shifted toward a hollow site. The next most favorable adsorption, with a binding strength of $2.67 \mathrm{eV}$, is when the radical is upright above the hollow site, regardless of the azimuth of the phenyl ring plane. The upright attachment at the bridge site has been found to be metastable with regard to either tilting toward the flat adsorbed radical or to displacement of the $\mathrm{S}$ atom to the hollow site. This site corresponds to a saddle point on the potential-energy surface. Constrained optimization results in a binding energy of $2.26 \mathrm{eV}$. In contrast

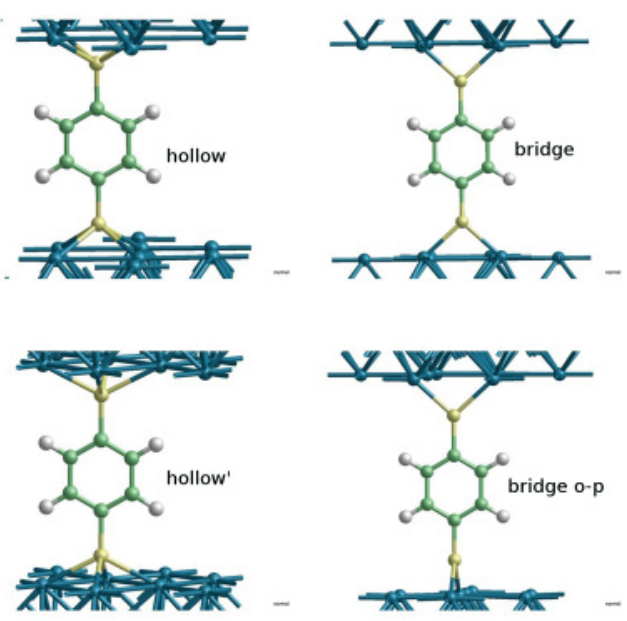

FIG. 4. (Color online) The geometries of hollow, hollow', bridge, and bridge $o-p$ adsorbed $1 \mathrm{R}$. 



FIG. 5. (Color online) Upper panel: Current vs bias voltage for the $1 \mathrm{R}$ wire in the five junction geometries between the $\mathrm{Au}(001)$ terminals. Lower panels: The zero-voltage transmission probability across these junctions. The energy is given relative to the Fermi level.

to the bridge and hollow sites, atop coordination is always found to be unstable and energetically the least favorable mode of binding. The potential-energy surface of PT on $\mathrm{Au}(001)$ thus exhibits features that are qualitatively very similar to those found previously in the study of PT/Au(111), ${ }^{7}$ despite the different methodology employed therein.

Upright bridge and hollow PT/Au(001) adsorption structures were then exploited to investigate the transport through the prototypical molecular wire PDT (1R). High-symmetry bridge adsorption between the two terminals can take place in three ways, depending on the angles that the two planes, defined by the three atoms involved in the surface bonds ( $\mathrm{Au}-\mathrm{S}-\mathrm{Au})$ on each terminal, make with the ring plane. In the first case, termed simply bridge, the three planes are parallel. In the second case, termed bridge o-o, the two planes of the coordinating atoms are orthogonal to the ring. Finally, in the third case, termed bridge $o-p$, the plane of the bridge site on the lower (upper) terminal is orthogonal (parallel) to the ring. High-symmetry adsorption on fourfold hollow sites can take place in two ways. In one case, labeled hollow, the ring plane is parallel to two sides of the Au squares (and perpendicular to the other two sides) on both contacts. In the other case, termed hollow', the ring plane is parallel to the square diagonals of both contacts. The hollow, hollow', bridge, and bridge $o-p$ adsorption geometries are illustrated in Fig. 4. The $I-V$ characteristics and zero-bias transmission coefficients $T(E)$ are shown in Fig. 5. For the hollow adsorbed 1R between the $\mathrm{Au}(001)$ terminals, the results are qualitatively similar to those obtained for the (111) orientation. The principal contribution to low-bias transport arises from the broad peak structure just below the Fermi level. The results for the hollow' geometry closely follow those of the plain hollow geometry, with a slightly more subdued transmission coefficient and, consequently, lower current. The three junctions with bridge adsorbed $1 \mathrm{R}$ exhibit higher conductances than the two with hollow adsorbed wires in the low- and moderate-bias range. The attachment via the energetically less-favorable bridge sites increases the energy of electronic states that give rise to the conducting channels, which is evident from the contribution to the PDOS (not shown) on the sulfur and carbon atoms. Hence, for the bridge adsorbed 1R, the Fermi level is located in the middle of the broad peak, rather than at its decaying tail, as in the junctions with hollow adsorbed 1R. The highest conductance is observed for the bridge o- $p$ geometry, for which a peak in the transmission spectrum coincides with the Fermi level.

\section{Conductance of nanoribbons between $\mathrm{Au}(001)$ terminals}

The $I-V$ characteristics and zero-bias transmission coefficients for nanoribbons from the 4-7-10-13R oligopyrene class



FIG. 6. (Color online) Upper panel: Current vs bias voltage for the 1-4-7-10-13R series of wires between the $\mathrm{Au}(001)$ terminals. Lower panels: The zero-voltage transmission probability across these junctions. The energy is given relative to the Fermi level. 
are shown in Fig. 6, together with those for the 1R structure. Again, the familiar trend of decreasing conductance with the length of the wire is reproduced, although in this case it is very weak. Similarly, the current peak magnitudes follow the same trend. However, an anomaly is observed for $1 \mathrm{R}$, which has been found to have less conductance than $4 \mathrm{R}$ and the three longer nanoribbons. This is a rather unexpected finding. It implies that for certain modes of terminal coupling and associated band alignment, an extended network of fused phenyl rings with multiple transport pathways across the backbone has potential benefits, which can more than compensate for the undesirable length effects.

The $I-V$ characteristics and zero-bias transmission coefficients for nanoribbons from the 5-8-11R oligoperylene class are shown in Fig. 7, together with those for the 2R structure. Two important observations can be made. First, the current through all the junctions is practically linear with the bias and, once again, it shows very little variation in magnitude between the wires. Hence, it appears as though the main source of resistance is at the contacts, while the wire length has almost no effect. Second, the transmission coefficients show a continuum of channels with high transmission probabilities over a large energy range. The only exception is the development of a gap close to $3 \mathrm{eV}$ for the 5,8 , and $11 \mathrm{R}$ wires, which would produce a lower current in the unlikely scenario of a bias in excess of $5 \mathrm{~V}$ being applied. The more flat and continuous structure of

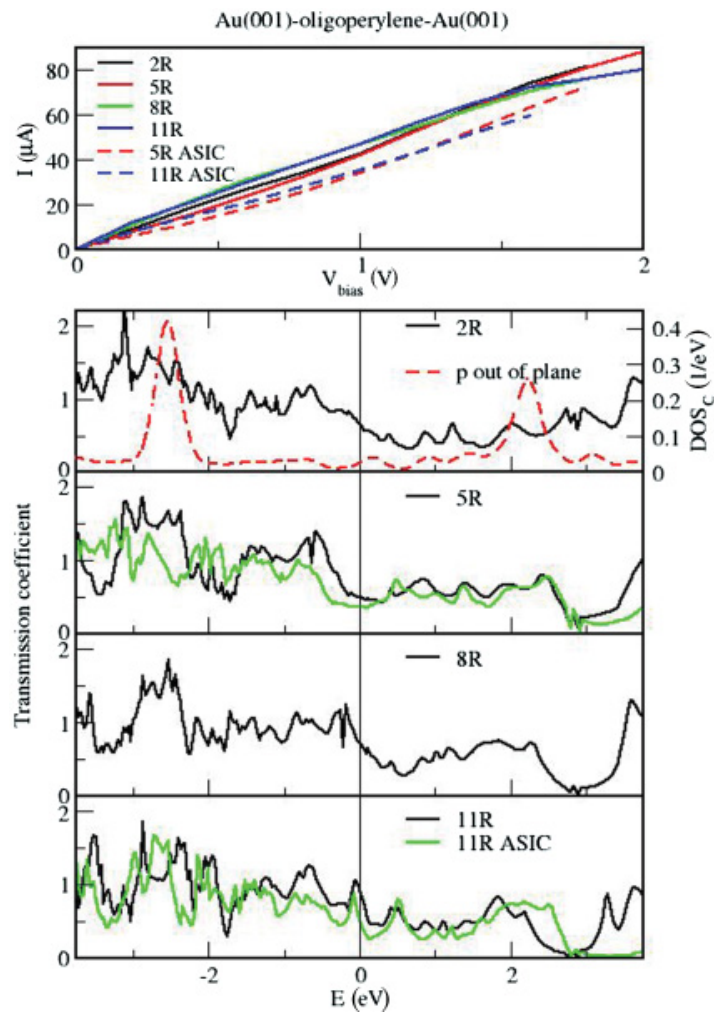

FIG. 7. (Color online) Upper panel: Current vs bias voltage for the 2-5-8-11R series of wires between the $\mathrm{Au}(001)$ terminals. Lower panels: The zero-voltage transmission probability across these junctions. The out-of-plane $p$ orbital contribution to the partial density of states (PDOS) from an edge $\mathrm{C}$ atom is also shown for the $2 \mathrm{R}$ wire in the lower panels. The energy is given relative to the Fermi level. the transmission spectra in the (001) junction, as opposed to the more discrete spectra of the (111) junction, is the consequence of the difference in the surface electronic structure. Frontier electronic states of the (001) junction display a higher degree of dispersion away from the zone center than their (111) counterparts. Hence, the peaks in the transmission spectra do not necessarily coincide with those in the PDOS. This is evident from the PDOS on the same $\mathrm{C}$ atom of the $2 \mathrm{R}$ wire in the (001) junction, also shown in Fig. 7. The ASIC results for the $5 \mathrm{R}$ and $11 \mathrm{R}$ wires in Fig. 7 show a very similar effect of the correction on the conductance as in the (111) junction. Even though the correction causes a similar reduction in the current, the $I-V$ characteristic remains almost linear and the conductance is still very high. The zero-bias transmission spectra evaluated with the ASIC exhibit somewhat lower amplitudes around the Fermi level, but their key feature, i.e., the broad continuum of highly conductive channels, still persists. Owing to the continuum of conducting channels in their spectra, both wires retain their linear characteristics in their $I-V$ curves. On the basis of the above findings, one can realistically expect high conductance from this family of nanoribbons, and little variation with the length of wire or bias. The present results are valid in the low-temperature limit, where the transport is essentially ballistic, without inelastic scattering and phase decoherence. Similar findings have been observed in short (up to $200 \mathrm{~nm}$ ) single-wall carbon nanotubes (SWNTs), where, for low biases, the conductance is length independent, indicating ballistic transport. ${ }^{22,23}$ Linear currentvoltage characteristics have been measured in both short SWNTs and narrow graphene nanoribbons. ${ }^{22,24}$ For longer molecular wires and elevated temperatures, phonon scattering is expected to significantly reduce the conductance. ${ }^{25}$

\section{Discussion}

The previous studies concerned with $4 \mathrm{R}$ and related crossconjugated wires focused on the pathway continuity of the HOMO and LUMO across the junction. ${ }^{1,2}$ Cohen et al. ${ }^{1}$ concluded that the lack of orbital density of those on the terminal $\mathrm{S}$ atoms is responsible for a lower-than-expected conductivity through $4 \mathrm{R}$, despite the favorable band alignment with the Fermi level of the leads and excellent degree of conjugation across the carbon backbone. Orbital plots of the HOMO and LUMO, shown in Fig. 10 of Ref. 1 and Fig. 7 of Ref. 2, demonstrate that due to the antisymmetry of those orbitals, not only the $\mathrm{S}$ atoms but also the central C-C atoms carry zero density. In fact, even the oligomers, such as $7 \mathrm{R}$, display the very same feature, as shown in Fig. 5(a) of Ref. 3. Hence, one could not expect that these orbitals would make a large contribution to the transport. The $\Gamma$-point state shown in Fig. 8 is an example of such an orbital for the $4 \mathrm{R}$ wire between the (111) and (001) terminals, respectively. In the plots of energy bands, also shown in Fig. 8, the orbitals that exhibit the left-right antisymmetry at the zone center often manifest themselves as straight lines, without dispersion, because they are decoupled from the gold contacts. Nevertheless, Liu et $a$. $^{2,3}$ suggested that the transport through oligopyrenes, such as 4-11R, follows a single pathway, which involves alternate single-double bonds along one of the edges and the central C-C atoms. Apart from the problem of zero HOMO- 

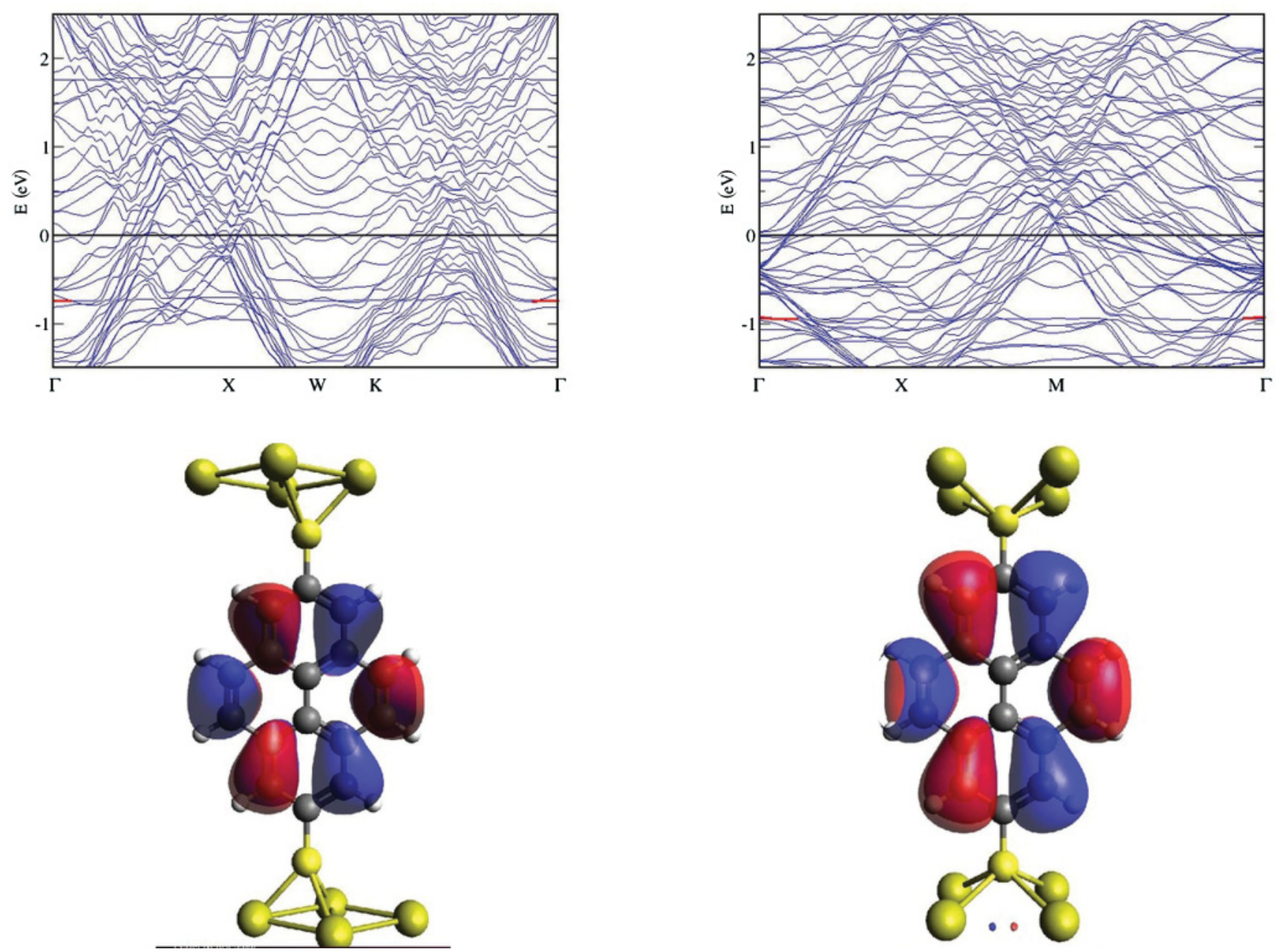

FIG. 8. (Color online) Upper panels: Energy bands of the electronic states in the junctions comprising the $4 \mathrm{R}$ wire between the Au(111) (left) and $\mathrm{Au}(001)$ (right) terminals. Only the states in the vicinity of the Fermi level are shown. The dispersionless occupied state, corresponding to the HOMO in the gas phase, is highlighted in red around the zone center. Lower panels: The orbital shape of this state at the zone center in the $\mathrm{Au}(111)$-based (left) and $\mathrm{Au}(001)$-based (right) junctions. Only the Au atoms forming the hollow sites are shown.

LUMO weights on the latter atoms, another problem with this interpretation is that the other edge, which is symmetric and equivalent, would not be conducting, despite the equally large contribution to the orbital density as the opposite, supposedly conducting, edge. The main rationale, apparently, for the single-pathway interpretation was the exponential dependence of the conductance of oligopyrene with length, which was similar to that observed for wires with a single conjugated chain, such as alkenes and oligophenyls. Interestingly, both Cohen et al. ${ }^{1}$ and Liu et $a l .{ }^{2}$ also seemingly overlooked the fact that the HOMO is nearly degenerate with a second HOMO (SHOMO). This is evident from the energy-level spectrum shown in Fig. 8(a) of Ref. 1. Unlike the HOMO and LUMO, the SHOMO is symmetric with respect to the long axis, resulting in a high orbital density on all the central as well as edge atoms. The states with this symmetry are all strongly coupled to gold, exhibit substantial dispersion along the k-points, and contribute most to the transmission channels. Therefore, we conclude that the single-pathway interpretation of transport in oligopyrenes is inappropriate and not supported by the facts. In fact, if one of the two middle rings becomes opened by the removal of the two edge $\mathrm{C}$ atoms, the results in Fig. 8(a) of Ref. 2 show a clear drop in the corresponding current.

Despite the differences in the interpretation, all the studies (including this work) have pointed out the relatively inefficient use of the multiple conjugated pathways potentially available to the electrons. This suggest that the main bottlenecks to the charge flow and sources of resistance are the contact points. In order to circumvent this problem, we propose the use of multiple terminal points, such as those in the $2 \mathrm{R}$ wire and oligoperylene series 5-11R. There are multiple benefits of this design. First, the multiple pathway continuity can be efficiently realized across the entire junction, i.e., not only across the carbon backbone. Second, the dual thiolate head groups provide a much stronger coupling between the terminals and carbon backbone In addition, in the more electron-rich oligoperylenes, additional frontier MOs with the out-of-plane $\pi$ symmetry are created, owing to the extra valent electrons compared to the oligopyrenes of the same length. Consequently, the HOMO-LUMO gap is much lower in the oligoperylenes than in the oligopyrenes, as illustrated in Fig. 9. Hence, for the former, the benefit of additional $\pi$-type 


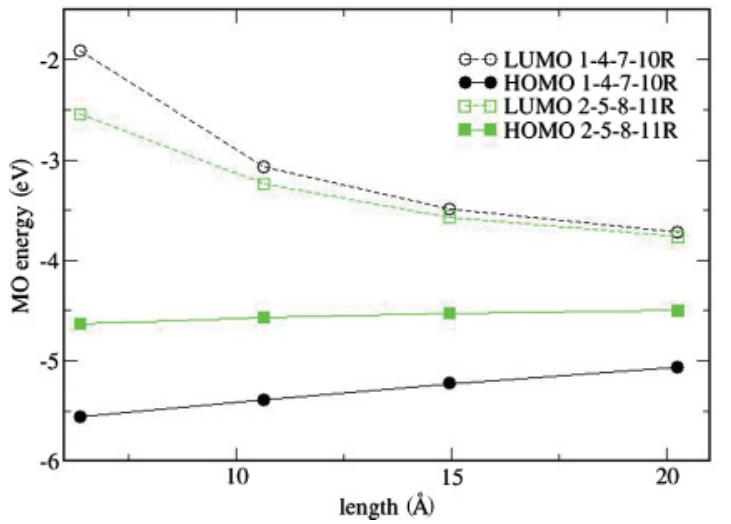

FIG. 9. (Color online) The HOMO and LUMO energy levels for 1-4-7-10R and 2-5-8-11R series of nanoribbons calculated with SIESTA.

frontier MOs in a decreasing energy band fully compensates for the penalty from having the longer wire. Finally, a less obvious benefit of the dual contact groups for the $2 \mathrm{R}$ junction is illustrated in Fig. 10, which shows five occupied and three unoccupied electronic states with $\pi$ symmetry at the zone center near the Fermi level. Two of the unoccupied states are antisymmetric with respect to the axis. Their orbital shapes correspond exactly to those of the HOMO and LUMO of the free molecule (with $\mathrm{H}$ atoms restored). While the orbital density on the central C-C atoms is zero, the dual thiolate groups on both sides of the junction carry a large orbital weight and are rather strongly coupled to the terminals. Hence, unlike their oligopyrene counterparts, they give rise to additional transmission channels via the two edge $\mathrm{C}-\mathrm{C}$ bonds.

\section{E. Effects of edge substitutions}

Finally, given the very narrow profile of all the nanoribbons considered, it is interesting to discuss the effects of the side edges on the overall performance of the wires. In the case of single-wall carbon nanotubes, for example, the hydrogen-passivated edges are known to influence the local electronic density and reactivity ${ }^{26}$ of the nanotube. Hydrogenated edges are the most common form of polycyclic aromatics, but fluorine is a possible alternative, and so are methyl $\left(-\mathrm{CH}_{3}\right)$ groups. While hydrogen typically donates a fraction of its charge to the coordinating carbon atom, fluorine is a strong electron acceptor, and methyl groups provide an approximation to a laterally extended carbon network as in graphene (however, the methyl $\mathrm{C}$ atoms are not aromatic). The $2 \mathrm{R}$ and $8 \mathrm{R}$ wires from the oligoperylene series of nanoribbons have been considered in the fluorinated form. In both cases, the $I-V$ characteristics remain nearly linear, but with a small conductance drop for the former and a quite large conductance drop for the latter. The corresponding transmission spectra (not shown) display a drop in the magnitude on the occupied-state side, which was not completely compensated for by an increase from the unoccupied-state side. The $2 \mathrm{R}$ wire has also been considered in the methylated form. In this case, an increase in the transmission appears on the occupied side, followed by a decrease on the unoccupied side. This results in a current still lower than that of the fluorinated wire. Hence, for


FIG. 10. (Color online) The shape of the electronic states with the out-of-plane $\pi$ symmetry and energy close to the Fermi level at the $\Gamma$ point for the $2 \mathrm{R}$ junction. The energies are given in $\mathrm{eV}$, relative to the Fermi level.

the best performing nanoribbons with dual terminal groups, conventional hydrogen edge termination seems to be the best choice. For the 4R wire from the oligopyrene set, fluorination slightly increased the conductance because of a small shift of the peak in the transmission above the Fermi level toward lower energies. However, methylation again caused a large drop of conductance. Overall, these calculations show that the simplest nanoribbons with hydrogenated edges perform best.

\section{CONCLUSIONS}

The conductance of two sets of aromatic nanoribbons has been modeled between gold contacts using the NEGF approach combined with DFT calculations. The effects of molecule length, multiple terminal thiolate groups, side edges, and symmetric pathways across the junction on charge transport have been investigated. The increase in the wire length has 
been found to cause a reduction in the conductance of the oligopyrene class of nanoribbons, with single thiolate groups at both terminals. For the oligoperylene nanoribbons, there was no evident length dependence, indicating that in the low-temperature limit of ballistic transport, the contacts are the key source of the resistance. In particular, single thiolate groups on the terminals have been predicted to present a bottleneck to the charge flow. In order to reduce the contact resistance and fully harness the multiple pathways available across the molecule backbone, dual thiolate groups at both terminals have been proposed. There are multiple benefits of this design. First, the multiple pathway continuity can be efficiently utilized across the entire junction, i.e., not only across the carbon backbone. Second, in the more electron-rich oligoperylenes, additional frontier MOs with the out-of-plane $\pi$ symmetry are formed, owing to the extra valence electrons compared to the oligopyrenes of the same length. Accordingly, the HOMO-LUMO gap is notably lower in the oligoperylenes than in the oligopyrenes. Finally, the frontier MOs, which are antisymmetric with zero orbital density on the central atoms, carry large orbital densities on the dual thiolate groups. Therefore, the dual terminal groups give rise to additional transmission channels and also provide a much stronger coupling between the terminals and carbon backbone. The use of $\mathrm{Au}(001)$ terminals provides an additional benefit as they give rise to the frontier electronic states with a high degree of dispersion. As a result, the transmission spectrum exhibits a continuum of channels and a very high conductance, practically independent of the bias or wire length. On the basis of the current findings, oligoperylene nanoribbons with dual terminal groups are expected to have considerable potential for use in molecular electronics.

\section{ACKNOWLEDGMENTS}

This work was supported by the Flexible Electronics Theme of the CSIRO Future Manufacturing Flagship. A.B. thanks CSIRO for support through the Julius Career Award program. J.D.G. thanks the ARC for funding under the Discovery scheme. The use of the NCI National Facility supercomputers at the ANU is gratefully acknowledged. The SMEAGOL project is sponsored by the Science Foundation of Ireland (Grant No. 07/IN/I945), by KAUST (Project No. FIC/2010/08), and by CRANN. *ante.bilic@csiro.au

${ }^{1}$ R. Cohen, K. Stokbro, J. M. L. Martin, and M. A. Ratner, J. Phys. Chem. C 111, 14893 (2007).

${ }^{2}$ H. Liu, W. Ni, J. Zhao, N. Wang, Y. Guo, T. Taketsugu, M. Kiguchi, and K. Murakoshi, J. Chem. Phys. 130, 244501 (2009).

${ }^{3}$ H. Liu, C. Yu, N. Gao, and J. Zhao, ChemPhysChem. 11, 1895 (2010).

${ }^{4}$ A. Bilić, Ž. Crljen, B. Gumhalter, J. D. Gale, I. Rungger, and S. Sanvito, Phys. Rev. B 81, 155101 (2010).

${ }^{5}$ A. K. Geim and K. S. Novoselov, Nature Mater. 6, 183 (2007).

${ }^{6}$ D. M. Ceperley and B. J. Alder, Phys. Rev. Lett. 45, 566 (1980).

${ }^{7}$ A. Bilić, J. R. Reimers, and N. S. Hush, J. Chem. Phys. 122, 094708 (2005).

${ }^{8}$ J. R. Reimers, Z. L. Cai, A. Bilić, and N. S. Hush, Ann. NY Acad. Sci. 1006, 235 (2003).

${ }^{9}$ J. R. Reimers, G. C. Solomon, A. Gagliardi, A. Bilić, N. S. Hush, T. Frauenheim, A. D. Carlo, and A. Pecchia, J. Phys. Chem. A 111, 5692 (2007).

${ }^{10}$ C. Toher, A. Filippetti, S. Sanvito, and K. Burke, Phys. Rev. Lett. 95, 146402 (2005).

${ }^{11}$ C. Toher and S. Sanvito, Phys. Rev. Lett. 99, 056801 (2007).

${ }^{12}$ C. Toher and S. Sanvito, Phys. Rev. B 77, 155402 (2008).

${ }^{13}$ C. D. Pemmaraju, T. Archer, D. Sánchez-Portal, and S. Sanvito, Phys. Rev. B 75, 045101 (2007).
${ }^{14}$ J. M. Soler, E. Artacho, J. D. Gale, A. García, J. Junquera, P. Ordejón, and D. Sánchez-Portal, J. Phys. Condens. Matter 14, 2745 (2002).

${ }^{15}$ L. Kleinman and D. M. Bylander, Phys. Rev. Lett. 48, 1425 (1982).

${ }^{16}$ N. Troullier and J. L. Martins, Phys. Rev. B 43, 1993 (1991).

${ }^{17}$ O. F. Sankey and D. J. Niklewski, Phys. Rev. B 40, 3979 (1989).

${ }^{18}$ J. Junquera, Ó. Paz, D. Sánchez-Portal, and E. Artacho, Phys. Rev. B 64, 235111 (2001).

${ }^{19}$ P. S. Damle, A. W. Ghosh, and S. Datta, in Molecular Nanoelectronics, edited by M. A. Reed and T. Lee (American Scientific, Los Angeles, 2003), p. 115.

${ }^{20}$ A. R. Rocha, V. M. Garcia-Suarez, S. W. Bailey, C. J. Lambert, J. Ferrer, and S. Sanvito, Phys. Rev. B 73, 085414 (2006).

${ }^{21}$ I. Rungger and S. Sanvito, Phys. Rev. B 78, 035407 (2008).

${ }^{22}$ J.-Y. Park, S. Rosenblatt, Y. Yaish, V. Sazonova, H. Üstünel, S. Braig, T. Arias, P. Brouwer, and P. McEuen, Nano Lett. 4, 517 (2004).

${ }^{23}$ C. Gomez-Navarro, P. D. Pablo, J. Gomez-Herrero, B. Biel, F. Garcia-Vidal, A. Rubio, and F. Flores, Nature Mater. 4, 534 (2005).

${ }^{24}$ G. Xu, C. T. Jr., J. Tang, J. Bai, E. Song, Y. Huang, X. Duan, Y. Zhang, and K. L. Wang, Nano Lett. 11, 1082 (2011).

${ }^{25}$ G. C. Solomon, A. Gagliardi, A. Pecchia, T. Frauenheim, A. D. Carlo, J. R. Reimers, and N. S. Hush, J. Chem. Phys. 124, 094704 (2007).

${ }^{26}$ A. Bilić and J. D. Gale, J. Phys. Chem. C 112, 12568 (2008). 